Revista de la Escuela de Ciencias de la Educación, año 15, NRO. 14, vol. 2, JUlio a diciembre de 2019. PÁGinas 81-89. ISSN 1851-6297 (DESDE DICIEMBRE DE 2006 A DICIEMBRE DE 2017). ISSN 2362-3349 (EN LíNEA). EVALUACIÓN DOCENTE Y DESEMPEÑO PROFESIONAL: EN JUEGO LA condición afectiva del profesorado. Mario Alberto Gallardo Bonilla. Carolina Pacheco Sosa.

\title{
EVALUACIÓN DOCENTE Y DESEMPEÑO PROFESIONAL: EN JUEGO LA CONDICIÓN AFECTIVA DEL PROFESORADO
}

\author{
Mario Alberto Gallardo Bonilla* \\ Benemérita Universidad Autónoma de Puebla, México \\ gallardobonillamarioalberto@gmail.com \\ Carolina Pacheco Sosa* \\ Benemérita Universidad Autónoma de Puebla, México \\ carophst@gmail.com
}

Recibido: 24/10/2018 Aceptado: 8/02/2019

\section{Resumen}

El presente trabajo evoca una de las necesidades del planteamiento de las políticas educativas actuales: la evaluación docente. El generar certeza de los desempeños a través de datos cuantificables. Así pues, se discute, más allá de desarrollar una evaluación objetiva, se plantea la idea de conocer qué tanto influyen los factores afectivos en el rendimiento del profesor.

En México se han puesto en marcha diversos instrumentos para una mejora educativa, los cuales parecieran más de corte administrativo que vinculados con el aspecto de capacitación personal. El presente trabajo desarrolla la idea de plantear una postura más humanista sobre los procesos. Se abordan entonces las variables afectivas en el nuevo sistema de evaluación al magisterio. De ahí que se proponga una metodología basada en el Modelo de Diseño Instruccional. Para determinar la relación existente entre los factores afectivos, ya no centrados en el estudiante, sino en el profesor, permiten un mejor desempeño profesional.

Palabras clave: Evaluación docente - Políticas educativas - Reforma educativa - Factores afectivos Desempeño docente.

\section{Abstract}

This research introduces the idea of the needs regarding current education policy: teacher's evaluation. The fact of promoting the idea of quantitative results is what the modern life proposes. As a matter of fact, this paper is focused on the idea, not to develop an accurate evaluation, but to recognize the idea of the importance of the affective factors at the moment of answering a teacher evaluation.

\footnotetext{
* Licenciado en lenguas modernas por la Benemérita Universidad Autónoma de Puebla, BUAP México. Cuenta con posgrado de maestría en Pedagogía por la Universidad Popular Autónoma del Estado de Puebla, UPAEP, México. Actualmente estudia el doctorado en Investigación e Innovación Educativa por la Benemérita Universidad Autónoma de Puebla, BUAP, México. Además, estudia la licenciatura en Derecho por la Universidad Nacional Autónoma de México, UNAM, México. Se encuentra adscrito a la Facultad de Lenguas como profesor de inglés en la Benemérita Universidad Autónoma de Puebla, BUAP, México.

* Licenciada en lenguas modernas por la Benemérita Universidad Autónoma de Puebla, BUAP México. Cuenta con posgrado de maestría en Pedagogía por la Universidad Popular Autónoma del Estado de Puebla, UPAEP, México. Actualmente realiza estudios en el Doctorado en Investigación e Innovación Educativa por la Benemérita Universidad Autónoma de Puebla, BUAP México. Colabora como docente de medio tiempo en la Secretaría de Educación Pública del Estado.
} 
Revista de la Escuela de Ciencias de la Educación, AÑo 15, NRO. 14, vol. 2, JUlio a diciembre de 2019. PÁGINAs 81-89. ISSN 1851-6297 (DESDE DICIEMBRE DE 2006 A DICIEMBRE DE 2017). ISSN 2362-3349 (EN LíNEA). EVALUACIÓN DOCENTE Y DESEMPEÑO PROFESIONAL: EN JUEGO LA condición afectiva del profesorado. Mario Alberto Gallardo Bonilla. Carolina Pacheco Sosa.

Mexico has developed a radical chance regarding educational politics. However, they seem to belong to the administrative field. It does not take into account several variables about teacher's needs.

This paper intends to develop a proposal based on the evaluation processes taking into account humanist aspects. These have been denied at the moment of presenting new politics. That is why it is developed a methodology to understand the affective factors, not focused on the student, but on the teacher. So that it can develop a better professional performance.

Key words: Teacher's evaluation - Educational politics - Affective factors - Teacher's performance Education legislation.

\section{Introducción}

Las políticas educativas en México han prevalecido sin mayores cambios, por lo menos hasta el último sexenio en que estas líneas toman parte. En el 2012, se llevaron a cabo reformas, incluido el ámbito educativo, las cuales impactan al quehacer docente de manera importante. Así, dichas reformas han influido directamente en nuestra carta magna por nombrar sólo una legislación, en adición y por consecuencia a la Ley General de Educación Federal y de cada entidad federativa, así como sus distintos reglamentos institucionales aunados a los sindicales.

Ahora bien, las reformas estructurales en materia educativa se reflejan hasta nuestros días, sin embargo, existen antecedentes no tan lejanos en los que podemos encontrar cambios significativos y sistemáticos en dicho ámbito. Por mencionar algunos, encontramos el sexenio del ex presidente de la República Ernesto Zedillo, en el cual se nos da cuenta de un cambio significativo en la manera en que se conforma un plan nacional de desarrollo. Se contó con el apoyo de investigadores y académicos de diversas instituciones para resarcir no sólo la problemática académica, sino también la decadencia y crisis económica en la que se encontraba el país en ese momento.

De este modo, se dio un aliento a la educación con el incremento del PIB dedicado a este rubro. En representación de las universidades públicas, en ese entonces el secretario general ejecutivo de la ANUIES, planteó entre otras cuestiones que la proporción del PIB destinada a la educación superior se incrementara a $1.5 \%$ ya que hasta entonces se asignaba un $57 \%$ del PIB nacional sin incluir el gasto destinado a la ciencia (Noriega, 2005). Lo anterior nos da muestra de un cambio significativo en la representación y peso de la educación para el gobierno federal.

Aunque se había tratado de implementar la política en el sexenio siguiente, el ahora ex presidente Fox había prometido un crecimiento mayor y sostenible. Sin embargo, el siguiente año presentó un panorama muy distinto, puesto que en la economía mexicana se empezaron a notar los efectos negativos producidos por la desaceleración económica de los Estado Unidos en razón de ser éste el mayor socio comercial de México (Díaz-Barriga y Mendoza, 2005).

Podemos ver que una reforma sustancial debía esperar por más tiempo debido a la inconveniencia económica del país, y es que dicha desaceleración mantuvo en un aletargamiento del país por casi tres años del sexenio, disminuyendo su crecimiento y postergando un cambio en la educación. Para el sexenio de Felipe Calderón, tenemos un cambio importante, la llamada "Alianza por la calidad de la educación" firmada por el mandatario del ejecutivo federal y la líder del Sindicato Nacional de Trabajadores de la Educación (SNTE), Elba Esther Gordillo. Dicho pacto pretendía elevar los niveles de la educación en México, sin embargo, tal y como lo menciona en su obra Ornelas (2012), el desencanto de dicho pacto se aprecia más como una especie de efecto Placebo que simplemente fungiera como una dicotomía entre la ilusión y la esperanza. El problema con la ambivalencia de la ACE es que hay contradicciones entre los fines que persigue. Una conjetura que se trabajó es que los propósitos manifiestos (elevar la calidad) se confunden con las intenciones implícitas (pacto electoral) y embrollan la ejecución (Ornelas, 2012). Ahora bien, como es sabido, el tema de la educación se plantea a los pocos días de la administración de Enrique Peña Nieto, Presidente de la República, dado que la reforma modifica lo que anteriormente se venía proclamando, y se limita el poder del sindicalismo a cuestiones de proteccionismo laboral. Y es que el sexenio comenzó con la realización del llamado Pacto por México, con el objetivo de aprobar lo que se llamaron como reformas estructurales, a saber: energética, educativa, político- electoral, telecomunicaciones, financiera, hacendaria y laboral (Núñez y Núñez de la Peña, 2016).

Con respecto a la educación y sus distintos cambios políticos acontecidos damos cuenta de la necesidad de estudiar el lado humano del docente, de sus necesidades, ambiciones y aspiraciones, así pues en la actualidad, nos encontramos con el concepto "motivación educativa" el cual es aplicado en diferentes ámbitos. Esto surge gracias a la necesidad que tienen las organizaciones de identificar cómo obtener el máximo provecho de sus colaboradores, y generar así, las condiciones necesarias que impulsen el desarrollo de la empresa.

El estudio de la motivación y los factores que la generan ha sido ampliamente expuestos en lo que se refiere a diversos ámbitos laborales. Sin embargo, cuando se enfoca al aspecto educativo, por lo general 
Revista de la Escuela de Ciencias de la Educación, año 15, NRO. 14, vol. 2, JUlio a diciembre de 2019. PÁGinas 81-89. ISSN 1851-6297 (DESDE DICIEMBRE DE 2006 A DICIEMBRE DE 2017). ISSN 2362-3349 (EN LíNEA). EVALUACIÓN DOCENTE Y DESEMPEÑO PROFESIONAL: EN JUEGO LA condición afectiva del profesorado. Mario Alberto Gallardo Bonilla. Carolina Pacheco Sosa.

dirige su atención a los modelos de enseñanza- aprendizaje y aquello que lleva a cabo el docente a impulsar a sus alumnos a que alcancen los estándares de desempeño necesarios para cumplir con el perfil de egreso.

Se ha llegado a la conclusión en este sentido, que los alumnos que se encuentran motivados evidentemente alcanzan resultados óptimos por encima de sus compañeros.

Partiendo de lo anterior, se pretende con el siguiente trabajo, mirar desde el otro lado del espejo a la motivación, pues se considera que se ha dejado de lado el interés por motivar a la planta docente, tanto desde el punto de vista directivo, como sistema educativo en general. Es por ello que buscamos dar respuesta a la manera en la que los maestros frente a grupo están siendo motivados por el sistema educativo, así como el impacto que esto genera en cuanto a la planeación, aplicación y evaluación de cada una de sus clases y las repercusiones que ello genera en sus alumnos.

Fue indispensable, a través de instrumentos de medición como: test actitudinales y de conocimientos, corroborar primero si existe una relación entre la motivación y el desempeño docente. De manera posterior, se analizaron los resultados y se llevaron a cabo diversas acciones para modificar los factores afectivos de tal modo que en un tercer momento de la investigación se pueda triangular dicha información. Con esto se pudo determinar si con dichas acciones se logró que la motivación funcionara como un elemento a favor del desempeño docente.

Así también, el presente trabajo tiene como fin último el dotar a la comunidad académica, específicamente la encargada del sector administrativo, de herramientas que le permitan tener un enfoque más participativo sobre los elementos que integran sus instituciones. En específico aquel que consideramos se le ha puesto como agente de responsabilidad, con respecto a los resultados en el proceso educativo. Sin embargo, poco se ha hecho para poder empoderarle de las herramientas para poder cumplir de manera más óptima con su desempeño docente.

Lo anterior debido a que el esfuerzo de los directivos de cada institución, lejos de enfocarse únicamente a obtener los mejores resultados de sus alumnos, previamente debería considerar optimizar el desempeño de los docentes, pues sólo así alcanzaría una cadena de mando que logrará mejorar las condiciones educativas de cada escuela.

\section{Contextualización}

La escuela Telesecundaria, comenzó a operar en el estado de Guanajuato México seis años después que el resto del país, en 1974. La subsecretaría de desarrollo educativo, es quien se encarga de vigilar que la modalidad lleve a cabo un funcionamiento óptimo a través del Programa de fortalecimiento a la Telesecundaria para el estado de Guanajuato.

Para el año 2015, y de acuerdo al catálogo educativo oficial de la Secretaría de Educación Pública del Estado de Guanajuato (2012), se encuentran activas un total de 1094 Telesecundarias en todo el estado. De las cuales 1087 son de sostenimiento público y 7 privadas.

La modalidad de Telesecundaria, tiene tal relevancia en el estado, que es quien mayor presencia de escuelas del nivel secundaria presenta, con un porcentaje de $55.57 \%$ de escuelas Telesecundarias.

En específico, la escuela Telesecundaria 1124 turno vespertino, comenzó a operar hace cuatro años, en el ciclo escolar 2012-2013, como parte del Programa de Fortalecimiento a la Telesecundaria para el estado de Guanajuato, siendo sede de la misma, las instalaciones de la Telesecundaria 581 turno matutino.

Debido a que es una escuela de aparente nueva creación, únicamente comparte los espacios que la Telesecundaria 581 ha destinado como tal, por lo que, en el turno vespertino, se cuenta con seis aulas, una para cada grupo; un área deportiva, plaza cívica, cuatro sanitarios, energía eléctrica, cisterna e internet.

Como ya se ha mencionado, la Telesecundaria se encuentra ubicada en la localidad de Ibarrilla, la cual está al norte del municipio de León en el estado de Guanajuato, constituye parte de la periferia de la ciudad, esto de acuerdo a los datos del Instituto Nacional De estadística y Geografía INEGI (2010), el suelo deja de ser llanura, para comenzar las estribaciones de la sierra de Guanajuato. Con un clima semiárido, subhúmedo con lluvias en verano de menor húmeda, cuenta con una población es de 20,000 habitantes.

Por su parte, el Informe Anual 2010 sobre la Situación de Pobreza y Rezago social, elaborado por la secretaría de Desarrollo Social (SEDESOL) y el Consejo Nacional de Evaluación de la política de Desarrollo Social (CONEVAL), arroja los siguientes resultados:

- $\quad$ En el municipio hay un $37.8 \%$ de pobreza, mismo del que se desprende la localidad, siendo parte de una pobreza moderada.

- Existen en Ibarrilla 798 habitantes de 15 años y más con educación básica incompleta.

- 650 personas están si derechohabientes a los servicios de salud.

Hablando del contexto económico de la comunidad, las personas se dedican a la albañilería, pespuntadores, mecánicos, obreros en fábricas de calzado, y comerciantes. Los principales problemas del contexto que afectan a la escuela están el pandillerismo, vandalismo, constantes robos en casas o en la calle, drogadicción, alcoholismo. 
Revista de la Escuela de Ciencias de la Educación, AÑo 15, NRO. 14, vol. 2, JUlio a diciembre de 2019. PÁGINAs 81-89. ISSN 1851-6297 (DESDE DICIEMBRE DE 2006 A DICIEMBRE DE 2017). ISSN 2362-3349 (EN LÍNEA). EVALUACIÓN DOCENTE Y DESEMPEÑO PROFESIONAL: EN JUEGO LA condición afectiva del profesorado. Mario Alberto Gallardo Bonilla. Carolina Pacheco Sosa.

\section{Marco teórico}

1. Motivación académica

La motivación se encuentra definida como los procesos que dan cuenta de la intensidad, dirección y persistencia del esfuerzo por conseguir una meta (Aldape, 2008). Lo que significa que de acuerdo a la intensidad con que se lleve a cabo una actividad habrá más posibilidades de conseguir o alcanzar una meta que haya sido establecida con antelación, siguiendo con palabras del autor, ésta indica que la motivación docente influye en el aprendizaje de sus alumnos, la motivación es un factor importante para lograr un alto desempeño, en la medida en que el docente se encuentre motivado crecerá su auténtico empeño en alcanzar la misión institucional (Aldape, 2008). De acuerdo a la autora, existe una inalienable relación entre los niveles motivacionales y el desempeño académico del docente, es decir que, si el docente se encuentra motivado, será capaz de desarrollar su labor ya no de manera eficiente, sino que ahora lo hará de manera eficaz, lo que en definitiva permeará en una administración laboral sana y más productiva. Ahora bien, la motivación en el aprendizaje es un elemento sustancial que ha de ser considerado por los diversos actores del ámbito académico, así pues, en el ámbito escolar, es unánime la consideración del papel fundamental que la motivación desempeña en la conducta académica (García, 2008). En el quehacer docente, debe existir una voluntad de hacer las cosas, y para que ésta exista primero que todo debe coexistir un elemento que permita a la persona empoderarse del deseo de hacer las cosas, para ello, en la educación, se debe preservar en el docente dicho elemento que al final incide en su desarrollo académico siempre y cuando se encuentre basado en el personal.

\section{Enfoque psicológico}

Para poder entender aún más a fondo el concepto de la motivación es importante tomar en cuenta y por razón de peso, el enfoque psicológico. Para lo anterior, primero que todo hay que entender la motivación como un proceso, el cual se encuentra constituido por necesidades, intereses y motivos (Abarca, 2002). De este modo, comprendemos que la motivación no es un fin, sino que es un medio para lograr un objetivo, y dicho medio debe de ser potenciado para que se pueda lograr un aumento en las posibilidades de que las metas propuestas puedan ser alcanzadas. En adición a lo anterior, se trata de optimizar los recursos con los que contamos, y si bien en el aspecto administrativo (de cualquier institución educativa) los recursos materiales cuentan, es preferible darles más énfasis a los recursos humanos con los que la escuela cuenta debido que son éstos últimos quienes son los únicos capaces de hacer operable cualquier industria, institución, corporación, entre otros. Otro aspecto que resulta del enfoque psicológico acerca de la motivación reside en saber que una persona no adquiere la motivación hacia algo como un acto individual, despojado de un contenido social. La motivación es también una expresión social y una construcción que toma sus ingredientes y sus contenidos de los procesos de interacción del sujeto con su medio, de un alumno con las tareas de aprendizaje, de un hijo con su entorno familiar, de un empleado con su práctica laboral (Abarca, 2002). Desde la perspectiva psicológica entendemos al hombre como un ser social, y es su entorno el que delimitará al sujeto con respecto a su motivación. El constructo social en que se encuentre será vital para que éste pueda desarrollarse pleno y sobre todo alcance sus metas de acuerdo a lo establecido para cada situación contextual en que se encuentre inmerso.

\section{Enfoque Conductual}

Rodríguez (1987) explica un estudio basado en la evolución, y parte del hecho de que los hombres al igual que los animales, poseen tendencias básicas que serán los móviles principales de la conducta. Los instintos son variables motivacionales primarias, innatas pero modificables, que proporcionan la energía esencial a la conducta humana. Son los que determinan y organizan todos los procesos mentales orientados hacia metas especiales. De lo anterior podemos observar que la motivación se limita a procesos mecánicos y primitivos del hombre, por lo que reduce a la motivación como un elemento fuera del proceso consciente. Es decir que, de acuerdo a la teoría conductista, la motivación no es consciente, sino que se lleva a cabo a partir de instintos animales de los cuales solamente existe un movimiento involuntario a los distintos refuerzos positivos o por algún reforzamiento negativo al que seamos expuestos, lo que significa que por dichos refuerzos son por los que el hombre subsiste. Así pues, se nos indica que los impulsos, en la tradición conductista se hallaban estrechamente ligados a las necesidades biológicas porque conducían al organismo a aprender cosas necesarias para sobrevivir (McClelland, 1989).

\section{Enfoque Cognitivo}

Para comprender el enfoque cognitivo nos remitiremos a Williams y Burden (1999), quienes afirman que el enfoque cognitivo colabora en la manera en que los individuos otorgan cierto sentido a sus experiencias al momento en que se lleva a cabo un aprendizaje, agregando que las personas se encuentran motivadas esencialmente por sus pensamientos, sentimientos y creencias. Así pues, podemos observar que, a diferencia del enfoque conductual, el cognitivo centra toda su atención en los factores afectivos y 
Revista de la Escuela de Ciencias de la Educación, año 15, NRO. 14, vol. 2, JUlio a diciembre de 2019. PÁGinas 81-89. ISSN 1851-6297 (DESDE DICIEMBRE DE 2006 A DICIEMBRE DE 2017). ISSN 2362-3349 (EN LÍNEA). EVALUACIÓN DOCENTE Y DESEMPEÑO PROFESIONAL: EN JUEGO LA condición afectiva del profesorado. Mario Alberto Gallardo Bonilla. Carolina Pacheco Sosa.

cognitivos, es decir que deja de lado un proceso mecánico y predecible del comportamiento humano. Para lo que incluye elementos que coadyuvan a que los procesos motivacionales cumplan el propósito de conseguir las metas establecidas. Pero no porque lo indique el instinto, sino por voluntad propia, porque así lo desea la persona y sobre todo porque así lo ha decidido, bajo un estado de conciencia que le permite llevar a cabo una toma de decisiones basadas en un raciocinio, más no por un estado primitivo de supervivencia.

\section{Enfoque Humanista}

En dicho enfoque se presentan variables que condicionan la motivación que se muestran aún más complejos. Se ha visto cómo se tratan los aspectos concernientes a la motivación desde un punto de vista primitivo, como lo hacen desde un punto de vista en que el raciocinio forma parte vital e inclusiva del hombre. Sin embargo, en el enfoque humanista existen elementos mencionados por Diaz-Barriga y Hernández (2002) tales como lo son las necesidades de libertad, la autoestima, el sentido de competencia capacidad de elección y autodeterminación. Para lo cual sus motivos centrales se encuentran guiados por el sentido de la autorrealización personal. Entonces, toda acción llevada por el hombre estará vinculada, no a satisfacer sólo sus necesidades primarias, y tampoco se limita a conocer, sino que se encuentra constituido por llevar a cabo una auto- realización personal, lograr metas que con respecto a los contextos sociales en que se encuentre inmerso, quiera lograr. Para ello, la persona se establecerá metas y se planteará objetivos que le permitirán seguir adelante pese a las vicisitudes que pudiese encontrar, todo lo anterior debido a la voluntad, la inteligencia, y la afectividad o emoción que dichos objetivos le proporcionen y que al final alimentarán su trascendencia. De este modo, el presente enfoque gira en torno a la persona como tal, exaltando así sus tipologías y características inherentes a la propia persona.

\section{Variables afectivas}

Existen diversas variables vinculadas al terreno de la afectividad que repercuten en el proceso de enseñanza-aprendizaje, y es que numerosas investigaciones en el campo educativo han intentado en los últimos 20 años obtener una mayor comprensión en relación con algunas variables afectivas que se relacionan con el rendimiento académico (Satelices, 1989). De este modo podemos comprender que existen investigaciones cuya base central se encuentra en la identificación de aquellas variables que posiblemente pudiesen concurrir como elementos vitales para que la motivación se lleve a cabo como una herramienta de apoyo para cumplir las demandas laborales o académicas. Sustento de lo anterior lo encontramos con Gardner y Lambert (1959) quienes afirman que más allá de las habilidades relacionadas al aspecto de la inteligencia verbal o aquella conocida como aptitud favorable, es indispensable poseer una variable socio afectiva positiva. Entonces, bajo esa condición, podemos observar que las variables afectivas cuentan como elementos que favorecen o incluso pueden limitar el desempeño de cualquier actividad, así como su rendimiento, tenemos previsto que suceda lo mismo el ámbito del desempeño docente. Es decir que sus capacidades cognitivas se vean mermadas por las distintas variables que en el siguiente apartado analizaremos más a profundidad.

\subsection{Ansiedad}

La ansiedad como una variable afectiva se muestra como un elemento invariable de todo ser vivo, desde las plantas, animales y los seres humanos sufrimos de dicha condición. Así, fruto de su utilidad para la supervivencia, la capacidad de generar ansiedad pasó a formar parte de nuestra esencia, a grabarse en nuestro código genético y transmitirse de generación en generación. Por lo anterior, no podemos dejar de sufrir ansiedad ya que somos humanos y los humanos nacemos preparados para experimentar ese tipo de respuesta (Caseras, 2009). Podemos observar que dicha condición es parte ya de nuestra existencia y por ella forma parte de nuestro contexto socio- cultural, para ello, es indispensable entonces, como todo ser vivo estar abierto a la posibilidad de adaptación del medio y de dicha respuesta al estímulo, precisamente del entorno. Para el autor líneas arriba mencionado, dicha condición establece la diferencia entre llevar a cabo un buen desempeño de las actividades personales y entre fracasar. En muchas ocasiones la gente que sufre de ansiedad percibe una amenaza sutil como la humillación de no rendir lo que los demás esperan de ellos. Es decir, sostienen la idea de que un buen profesional debe siempre responder de manera convincente y bien argumentada a una pregunta (Caseras, 2009). Sustentamos la idea sobre la presión otorgada al docente, quien en el aula no puede cometer un error, debido a que los estudiantes pueden ser incisivos y sobre todo audaces a la hora de corregir al profesor antes cualquier error que este cometa. En la educación pública, en el contexto mexicano hablamos de grupos que oscilan entre 40 a 50 estudiantes por aula en nivel básico, y por ende dicha presión será mayor en la medida en que el profesor cometa más errores. Su ansiedad podría aumentar considerablemente provocando aún más que dicha variable acentúe el problema cayendo así en un círculo vicioso que al final incidirá en un bajo desempeño profesional del docente y por ende de los estudiantes. 
Revista de la Escuela de Ciencias de la Educación, AÑo 15, NRO. 14, vol. 2, JUlio a diciembre de 2019. PÁGINAs 81-89. ISSN 1851-6297 (DESDE DICIEMBRE DE 2006 A DICIEMBRE DE 2017). ISSN 2362-3349 (EN LíNEA). EVALUACIÓN DOCENTE Y DESEMPEÑO PROFESIONAL: EN JUEGO LA condición afectiva del profesorado. Mario Alberto Gallardo Bonilla. Carolina Pacheco Sosa.

\subsection{Inhibición}

El tema de la inhibición como un sistema motivacional lo encontramos descrito por Chiclana y Contreras (2015) así pues, dicho apartado dedicado al estudio de la inhibición propone una teoría que cuenta con dos sistemas motivacionales. A saber, el primero es el de sistema de inhibición o evitación conductual y el sistema de activación o aproximación conductual. Ambos sistemas forman parte del mecanismo neuroconductual vinculado al esfuerzo y al castigo los cuales conforman la base de la personalidad. Es entonces indispensable que exista un balance entre dichos sistemas de modo tal que permita mantener un equilibrio para que el mecanismo denominado neuroconductual no desvirtúe la personalidad de quien se encuentre desempeñando determinada actividad. Ahora bien, nos enfocaremos en el sistema motivacional de inhibición, también conocido como SIC o BIS, el cual conforma un dispositivo de refuerzo negativo, que acciona ante señales consideradas aversivas, respondiendo así a estímulos de no recompensa o castigo (Chiclana y Contreras, 2015). En otras palabras, cuando la inhibición sucede nos damos cuenta de que toda conducta queda total o parcialmente suprimida ante cualquiera que sea el estímulo, lo que podría significar que prácticamente se evita la confrontación de dicho estímulo, ya sea que pueda ser positivo o en su defecto negativo, provocando en el individuo un aletargamiento en su capacidad de respuesta y desde luego en su desempeño, en el caso de un docente su desempeño profesional.

\section{Evaluación}

Evaluar, es un tema que hoy por hoy se concibe como parte del discurso educativo que nos rodea. Todo aquel que estemos involucrados en el actuar educativo, sabemos que la evaluación no es un tema aparte y que nos atañe a todos por igual.

Diferentes han sido las acepciones que se le han atribuido al concepto de evaluación, desde lo tradicionalista en el cual se concibe como una parte final de un proceso, hasta la concepción contemporánea,

La evaluación de los aprendizajes es un proceso constante de producción de información para la toma de decisiones, sobre la mejora de la calidad de la educación en un contexto humano social, mediante sus funciones diagnóstica, formativa y sumativa. (Segura, 2009, p.1).

Donde, de acuerdo al autor, la evaluación no concluye con un proceso, sino que lo acompaña desde el inicio, se va transformando, y permite identificar las acciones con las que se está actuando, determinando aquello que es de utilidad para alcanzar los objetivos propuestos, o bien, para indagar en aquellas áreas de oportunidad en las que es necesario redoblar esfuerzos. También, la evaluación se presenta en tres momentos específicos: al inicio, durante el proceso y al finalizarlo, con la intención de obtener un panorama general y tener un marco de referencia sobre los propósitos alcanzados con respecto al punto de partida.

\section{Examen de evaluación profesional}

La ley general del servicio profesional docente, publicada el 11 de septiembre de 2013, establece los parámetros bajo los cuales los docentes de educación básica y media superior deben regular sus acciones para poder contar con las condiciones de ingreso, promoción reconocimiento y permanencia en el servicio.

Es en el Título II, capítulo III, desde el artículo 21 al 25, donde se habla acerca del ingreso al servicio, y se menciona que este se llevará a cabo mediante concursos de oposición, los cuales buscarán que de acuerdo al nivel para el cual se postule, los docentes cuenten con los conocimientos y capacidades que la profesión le demande.

Los exámenes están divididos en dos áreas:

I. Conocimientos y Habilidades para la Práctica Docente: pretende identificar el nivel de dominio con el que cuentan los docentes acerca de los propósitos de cada asignatura o campo formativo. Así como las capacidades y habilidades que les permitirán llevar a cabo intervenciones pedagógicas y didácticas oportunas de acuerdo al nivel en donde se desenvuelven.

II. Habilidades Intelectuales y Responsabilidades Ético-Profesionales: Evalúa las habilidades con las que cuenta el docente en cuanto a la mejora de su práctica y que ésta a su vez genere un impacto en la comunidad donde labora, impulsando el desarrollo de la calidad educativa.

Una vez que el docente obtenga resultados satisfactorios y se le otorgue el nombramiento correspondiente, está en la obligación de acuerdo a la misma Ley, de presentar el examen de diagnóstico al término de su primer año de servicio en el Sistema Educativo Nacional.

Esta evaluación tiene como propósito identificar las fortalezas y oportunidades de los docentes con el fin de ofrecer apoyos en cuanto a las necesidades que requiera subsanar.

Este proceso consta de tres fases:

I. Notificación a docentes y técnico docentes del proceso de evaluación al cual estarán sujetos.

II. Aplicación de instrumentos de evaluación:

i. Reporte de cumplimiento de responsabilidades profesionales 
Revista de la Escuela de Ciencias de la Educación, año 15, NRO. 14, vol. 2, JUlio a diciembre de 2019. PÁGinas 81-89. ISSN 1851-6297 (DESDE DICIEMBRE DE 2006 A DICIEMBRE DE 2017). ISSN 2362-3349 (EN LíNEA). EVALUACIÓN DOCENTE Y DESEMPEÑO PROFESIONAL: EN JUEGO LA condición afectiva del profesorado. Mario Alberto Gallardo Bonilla. Carolina Pacheco Sosa.

ii. Examen de conocimientos y habilidades docentes que favorecen el aprendizaje de los alumnos

iii. Cuestionario de características y necesidades formativas de los docentes y técnicos docentes de nuevo ingreso

III. Entrega y difusión de resultados-emisión de informe personal.

En el capítulo IV del mismo título, donde se expresa que, para aspirar a las funciones de director o supervisor, también se debe llevar a cabo un proceso mediante un concurso de oposición, el cual se encarga de evaluar lo siguiente:

I. Conocimientos y Habilidades para la Práctica Docente: Evaluar el conocimiento de la organización y funcionamiento de la escuela

II. Habilidades Intelectuales y Responsabilidades Ético-Profesionales: Evaluar las habilidades del docente, en cuanto a gestión escolar, que le permita mejorar las condiciones educativas de las instituciones para las cuales labora.

El capítulo VIII, la permanencia en el servicio es otro tema en el que se centran las evaluaciones profesionales. Siendo así, se pretende identificar el nivel de desempeño de los docentes. De tal modo que, si los resultados llegaran a ser catalogados como insatisfactorios o insuficientes, se le pudiera asignar al docente la incorporación a programas de regularización y tutoría. Para posteriormente volver a sustentar la evaluación correspondiente, y en caso de que se vuelva a contar con resultados insatisfactorios después de tres oportunidades, se le dará por terminado su contrato con la Secretaría de Educación Pública.

Entonces, de acuerdo a los nuevos parámetros establecidos por la Ley General de Educación, y la Ley del Servicio Profesional docente, todo aquel que pretenda ingresar, promoverse o continuar en el Sistema Educativo Nacional Mexicano, debe ser forzosamente sometido a un proceso de evaluación que garantice que el personal cuente tanto con los conocimientos, pero a su vez las habilidades y actitudes que se necesitan para impulsar la calidad en la educación, dependiendo del nivel y la modalidad en la cual se desenvuelvan.

Bajo este código, queda obsoleta la acción de heredar plazas, o de obtener alguna mediante la vía sindical. Lo que se pretende, es que únicamente la persona "idónea" para la labor, sea quien permanezca en ella.

El contenido de la propuesta cuenta con dos evaluaciones, a saber; la primera de carácter cognitivo y la segunda de carácter afectivo para conocer los niveles de afectividad. En otro momento la propuesta consiste en proporcionar tres sesiones pertinentes relacionadas a la motivación (curso - taller, elaborado por especialistas en el área de psicología) acerca de elementos importantes para la persona y su desarrollo personal, académico y profesional. Dichos talleres serán de una duración de una hora cada uno y serán aplicados durante tres semanas respectivamente. La población a la que se encuentra dirigida es de seis docentes, de los cuales cinco son de género femenino y uno masculino. Sin embargo, es importante precisar que se sometieron al proceso de evaluación y de participación de los cursos- talleres cuatro docentes de género femenino, la edad promedio de los participantes es de 25 a 40 años.

La propuesta del presente trabajo práctico es llevar a cabo una evaluación de los factores afectivos, basada en escalas, la cual ha sido adaptada por Gardner (1982). Lo que pretendemos conocer es si los factores afectivos y emocionales influyen de manera directa con el desempeño del docente de educación básica al momento de llevar a cabo su labor docente. Por lo que será preciso identificar sí los profesores de educación básica se sienten desmotivados al momento de llevar a cabo su labor docente. Posteriormente, detectar sí con el aumento de incentivos motivacionales externos el desempeño del profesor de educación básica adopta alguna modificación con respecto a su labor académica.

\section{Metodología}

Consiste en tres etapas, a saber; la primera en la aplicación y análisis de las pruebas cognitivas y actitudinales, es decir la aplicación de dos cuestionarios concernientes a lo afectivo y al desempeño docente. En un segundo momento, la implementación de cursos- talleres motivacionales. En un tercer momento, una nueva aplicación de los mismos instrumentos junto con su análisis. Finalmente, un cuarto momento; la triangulación de la información de los resultados de la primera prueba con respecto de la segunda tomando como una variable la aplicación de los cursos- talleres para determinar si los factores afectivos tienen presencia en el desempeño docente.

\section{Modelo de Diseño instruccional}

Para poder llevar a cabo el trabajo práctico, se hace mención del modelo que se siguió para desarrollar los talleres implementados, el modelo: ADDIE (Análisis, Diseño, Desarrollo, Implementación, y Evaluación de los materiales de aprendizaje y las actividades), el cual consta de una serie de pasos interrelacionados que permiten la organización del sistema para generar un diseño instruccional (Steven, 2000). 
Revista de la Escuela de Ciencias de la Educación, AÑo 15, NRO. 14, vol. 2, JUlio a diciembre de 2019. PÁGINAs 81-89. ISSN 1851-6297 (DESDE DICIEMBRE DE 2006 A DICIEMBRE DE 2017). ISSN 2362-3349 (EN LíNEA). EVALUACIÓN DOCENTE Y DESEMPEÑO PROFESIONAL: EN JUEGO LA condición afectiva del profesorado. Mario Alberto Gallardo Bonilla. Carolina Pacheco Sosa.

El primer paso del modelo, consiste en el análisis de las personas a evaluar, el contexto en el cual se encuentran inmersas, así como las herramientas con las cuales se cuenta para poder llevar a cabo el resto del proceso. Es el antecedente que genera el problema, su origen, así como la elaboración tentativa de las posibles soluciones.

Esta parte del proceso fue aplicada mediante una entrevista a los docentes de la Telesecundaria 1124, en donde se les dio a conocer la propuesta, así como solicitar su apoyo a la misma, o en caso contrario, externar su negativa al respecto, con el afán de dar seguimiento a los siguientes pasos.

El diseño es la parte del proceso que sistematiza los resultados arrojados del análisis, para poder convertirlos en la planeación del contenido de la instrucción. Se lleva a cabo una planeación, que toma en cuenta ¿qué es lo que pretendemos alcanzar? (objetivos), ¿de qué manera? (actividades), así como el tiempo en el cual tenemos destinado alcanzar dichos objetivos, aunado a las estrategias, materiales, recursos y la evaluación.

En la fase del desarrollo, se le da vida a aquello planeado en el diseño, específicamente se generan los ejercicios a realizar, se preparan los materiales y recursos, y se impulsa la creación del ambiente de aprendizaje.

La implementación, consta de la aplicación del diseño instruccional con los docentes a los cuales va dirigido, y finalmente la evaluación reúne de manera sistemática la información arrojada después del proceso, para poder generar el informe pertinente y las recomendaciones para futuras aplicaciones.

De esta manera, se aplicaron las dos pruebas, la relacionada a las cuestiones de carácter profesional, dicha prueba se encuentra basada en las examinaciones que se realizan en el Servicio Profesional Docente y que determinan la idoneidad de cada profesor de acuerdo al nivel educativo en que éste se encuentre desempeñando sus funciones. Dicha prueba tiene una duración de 1 hora y consiste en el contenido conceptual sobre docencia. Posterior a dicha prueba se precisó completar el test con el contenido de las variables afectivas, el cual tuvo una duración de 25 minutos y cuenta con dos apartados. Los resultados fueron analizados y posterior a ello, se diseñó y desarrolló el taller con los maestros de la Telesecundaria 1124 durante la última semana del ciclo escolar 2015-2016 y las primeras semanas del ciclo escolar 2016- 2017, cuyo objetivo fue reforzar la motivación a la profesión docente, para que a un mes de haber aplicado la primera prueba, se apliquen nuevamente los dos instrumentos; tanto el examen de conocimientos como el test actitudinal y entonces triangular la información para poder determinar si las herramientas y el taller motivacional, coadyuvaron a que se mejorara del desempeño docente.

\section{Conclusiones}

Primero que todo, es importante mencionar que el presente trabajo ha corroborado que los factores afectivos juegan un papel preponderante en el desempeño docente y los profesores mejoraron de manera significativa. En primer lugar, pudimos identificar que los profesores de educación básica se sentían desmotivados al momento de llevar a cabo su labor docente. De acuerdo a la prueba actitudinal, se pudo corroborar dicha información, pues lo docentes no sentían satisfacción por su trabajo. En segundo lugar, pudimos detectar que con el aumento de incentivos motivacionales externos el desempeño del profesor de educación básica adoptó modificaciones y el resultado se observa en la segunda evaluación de carácter académico. En tercer lugar, a partir de Diseño Instruccional motivacional externo se pudo analizar la motivación en los profesores de educación básica. En adición, la pertinencia del trabajo dependerá de las instituciones, pues se requiere de disponibilidad por parte de directivos para que se pueda llevar a cabo. En cuanto a la viabilidad del Diseño Instruccional se considera eficaz para aplicarlo en diversos momentos. Esto para poder cubrir las necesidades, al tiempo de solicitar apoyo del personal del departamento psicopedagógico para su auxilio. Las limitaciones son, precisamente el no contar con un departamento que pueda brindar apoyo con respecto a los talleres y su aplicación.

Finalmente, se considera que para futuras investigaciones se puedan tomar en cuenta variables, tales como la cantidad poblacional a la que vaya dirigida, se sugieren sea mayor para determinar y verificar dichos resultados con una población mayor. Se recomienda su aplicación en instituciones de carácter privado ya que las circunstancias de trabajo entre una escuela pública y una privada varían. Además que se pueda llevar en un contexto urbano, pues como se mencionó, dicho trabajo fue llevado a cabo en un contexto rural.

\section{Referencias Bibliográficas}

- Abarca, S. (2002). Psicología de la Motivación. Costa Rica: Editorial Universidad Estatal a Distancia.

- Aldape, T. (2008). Desarrollo de Las Competencias del Docente. Demanda de La Aldea Global Siglo XXI. Libros en Red

- $\quad$ Barriga, A. y Mendoza J. (2005). Educación superior y Programa Nacional de Educación, 2001-2006: aporte para una discusión. México: Biblioteca de la Educación Superior.

- Chiclana, C. y Contreras, M. (2015). Trabajos Fin de Grado de Psicología. Selección 2011-2014. España: 
Revista de la Escuela de Ciencias de la Educación, año 15, NRO. 14, vol. 2, JULIO A diciembre de 2019. PÁGINAs 81-89. ISSN 1851-6297 (DESDE DICIEMBRE DE 2006 A DICIEMBRE DE 2017). ISSN 2362-3349 (EN LíNEA). EVALUACIÓN DOCENTE Y DESEMPEÑO PROFESIONAL: EN JUEGO LA condición afectiva del profesorado. Mario Alberto Gallardo Bonilla. Carolina Pacheco Sosa.

Biblioteca de Salud Mental.

- García, F. (2008) Motivar para el aprendizaje desde la actividad orientadora. España: Ministerio de Educación y Ciencia.

- Gardner, R. (1982). Language attitudes and language learning In E. Boudhard Ryan \& H. Giles, Attitudes towards language variation. Edward Arnold.

- INEGI. (2010). "Compendio de información geográfica municipal 2010 León”. Guanajuato. México: Instituto Nacional de Estadística y geografía.

- $\quad$ Noriega, M (2005). Cultura política y política educativa en el sexenio de Ernesto Zedillo. México: Plaza y Valdés editores.

- Núñez, J. y Núñez de la Peña, F. (2016). México se cimbra a la mitad del sexenio. Análisis Plural: México.

- Ornelas, C. (2012). Educación, Colonización y Rebeldía: La herencia del pacto Calderón- Gordillo. México: Siglo XXI.

- Rodríguez, R. (1987). Apuntes de la Psicología de la Motivación. España: Universidad de

- Oviedo.

- SEG. (2012). "Programa estatal de fortalecimiento de telesecundaria por el estado de Guanajuato 2012". Guanajuato. México: Secretaría de Educación del estado de Guanajuato.

- Segura, M. (2009). La evaluación de los aprendizajes basada en el desempeño por competencias. Revista Electrónica Actualidades investigativas en educación. Costa Rica.

- $\quad$ Steven J. (2000). Instructional Systems College of Education. Penn State University 